\title{
Strenuous Exercise-An Unusual Cause of Deranged Liver Enzymes
}

\author{
Nicholas-Paul Delicata ${ }^{1}$, Julian Delicata ${ }^{2}$, Lara-Anne Delicata ${ }^{2}$ \\ ${ }^{1}$ Institute of Liver Studies, King's College Hospital, London, UK \\ ${ }^{2}$ Department of Medicine, Mater Dei Hospital, Msida, Malta \\ Email: ^nicholas-paul.delicata@gov.mt
}

How to cite this paper: Delicata, N.-P. Delicata, J. and Delicata, L.-A. (2018) Strenuous Exercise-An Unusual Cause of Deranged Liver Enzymes. Case Reports in Clinical Medicine, 7, 177-181. https://doi.org/10.4236/crcm.2018.73016

Received: January 31, 2018

Accepted: March 4, 2018

Published: March 7, 2018

Copyright (c) 2018 by authors and Scientific Research Publishing Inc. This work is licensed under the Creative Commons Attribution International License (CC BY 4.0).

http://creativecommons.org/licenses/by/4.0/

\begin{abstract}
Liver enzymes and function tests are routinely taken in clinical practice. Deranged liver enzymes however, do not always necessarily imply an underlying liver pathology. The standard liver enzymes measured include alanine aminotransferase (ALT), aspartate aminotransferase (AST), gamma-glutamyltranspeptidase (GGT) and alanine phosphatase (ALP). These enzymes, especially ALT and AST, can be released by other organs in the body. We report an unusual case of a 48-year-old patient with deranged enzymes related exclusively to intensive exercise that resolved on discontinuing such exercise.
\end{abstract}

\section{Keywords}

Deranged Liver Enzymes, Alanine Aminotransferase, Aspartate Aminotransferase, Detailed History, Intensive Exercise

\section{Introduction}

A detailed history taking is paramount to establish a thorough differential diagnosis and to identify other potential causes when faced with deranged liver enzyme tests. This is of benefit because it might reduce the number of invasive tests, reduce expenses and limit the potential complications to the patient. We report the case of deranged liver enzymes in a 48-year-old male performing regular intensive exercise.

\section{Case Report}

A 48-year-old Asian male was referred to our liver clinic in view of deranged liver enzymes. The AST was $106 \mathrm{IU} / \mathrm{L}$ (normal range 10 - $50 \mathrm{IU} / \mathrm{L}$ ) and ALT was 66 IU/L ( 5 - 55 IU/L). The remaining liver enzymes (ALP and GGT) and bilirubin 
were within normal limits. Prothrombin time and albumin levels were also normal.

On history taking, there were no particular risk factors. He denied alcohol consumption, did not partake in recreational drugs and was a non-smoker. There was no family history of liver disease. On examination he was lean, with no stigmata of chronic liver disease.

An ultrasound showed a liver that was normal in shape, size and reflectivity with no focal lesions. The bile ducts were not dilated. The gallbladder was normal in shape and size with a normal wall thickness and no gallstones were seen. The portal vein was patent with antegrade flow and the spleen was unremarkable with a length of $9.8 \mathrm{~cm}$. No free fluid was present.

A chronic liver screen taken at time of presentation showed a negative auto-immune screen, normal serum copper levels, normal ferritin levels and a normal alpha-1 antitrypsin phenotype. The Hepatitis B surface-antigen (HbsAg), Hepatitis B core-antibody ( $\mathrm{HbcAb}$ ) and Hepatitis B e-antibody (HbeAb) were all positive with a Hepatitis B serum viral level of $662 \mathrm{IU} / \mathrm{ml}$. The remaining viral screen (Hepatitis A, Hepatitis C, Hepatitis D, Hepatitis E and Human Immunodeficiency Virus) was all negative, indicating no current or previous exposure to any of these viruses.

The presumed diagnosis was a transaminitis secondary to Hepatitis B infection. A FibroScan ${ }^{\circledast}$ showed a liver stiffness of $4.2 \mathrm{kPa}(2-7 \mathrm{kPa})$ and a controlled attenuation parameter (CAP) of $252 \mathrm{~dB} / \mathrm{m}(100-400 \mathrm{~dB} / \mathrm{m})$.

In view of the relatively low enzyme levels and the low viral replicating levels, with no evidence of liver damage and no family history of liver disease, he was not started on anti-viral treatment. Over the following few months the liver enzymes remained persistently deranged with high AST and ALT levels despite the low levels of Hepatitis B viral DNA in serum (296 IU/ml) (Figure 1).

ALT and AST correlated with HBV DNA over 3 years

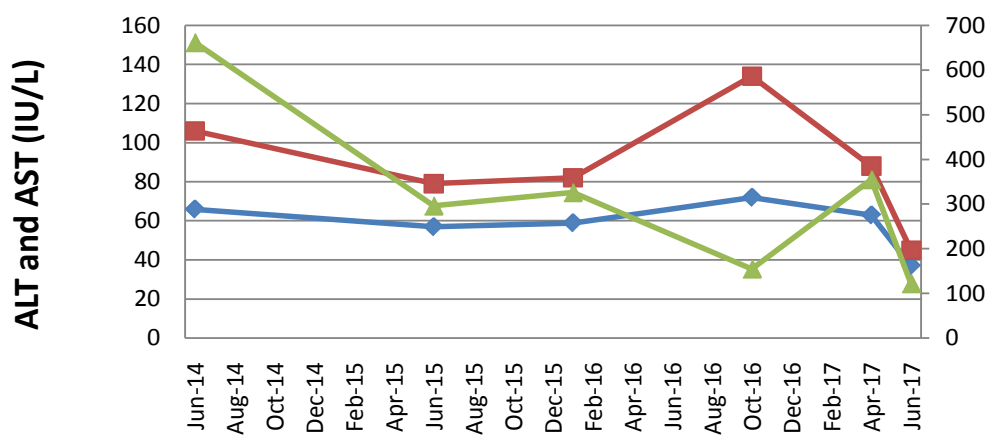

\begin{tabular}{|l|c|c|c|c|c|c|}
\cline { 2 - 7 } \multicolumn{1}{c|}{} & Jun-14 & Jun-15 & Jan-16 & Oct-16 & Apr-17 & Jun-17 \\
\hline$\sim$ - ALT (IU/L) & 66 & 57 & 59 & 72 & 63 & 37 \\
\hline- AST (IU/L) & 106 & 79 & 82 & 134 & 88 & 45 \\
\hline$\sim$ HBV DNA (IU/mL) & 662 & 296 & 326 & 155 & 355 & 122 \\
\hline
\end{tabular}

Figure 1. Correlation of ALT and AST with HBV DNA over a 3-year period. 
In view of these results a liver biopsy was organized and subsequent histology analysis only showed mild portal fibrosis and minimal inflammatory activity. The changes in this sample were minimal and non-specific and were not typical of an active, chronic hepatitis B infection.

In the absence of evident ongoing liver damage, treatment was again not initiated. On delving further into our patient's history and daily activities, he reported intensive exercise training regimes six times a week with only one rest day. A creatine kinase (CK) was thus taken and was raised at $860 \mathrm{IU} / \mathrm{L}(<150$ IU/L). Subsequently the CK was monitored closely together with the AST and ALT (Figure 2).

Since there was now a strong possibility that these changes in liver enzymes could be related to exercise, he was advised to stop training for a few weeks. Following this period of abstinence from his regular intense exercise regimen, both the AST and ALT normalised (45 IU/L and $37 \mathrm{IU} / \mathrm{L}$ respectively). The CK was $40 \mathrm{IU} / \mathrm{L}(<150 \mathrm{IU} / \mathrm{L})$. This strong correlation in the blood results pointed to the exercise regimes being the likely causative aetiology for the deranged enzymes, making the diagnosis of Hepatitis B an incidental, though important, finding.

\section{Discussion}

Our patient was referred with deranged blood tests and was asymptomatic. The widespread availability of testing liver profile and liver synthetic function has increased the pick-up rate of liver disease. It is this increased availability that emphasises the importance of taking a detailed history from the patient in the first instance. A routine check-up for our patient took him down a diagnostic pathway, which included a number of investigations, both invasive and non-invasive.

Whilst an important diagnosis of chronic hepatitis B with low viral replicating

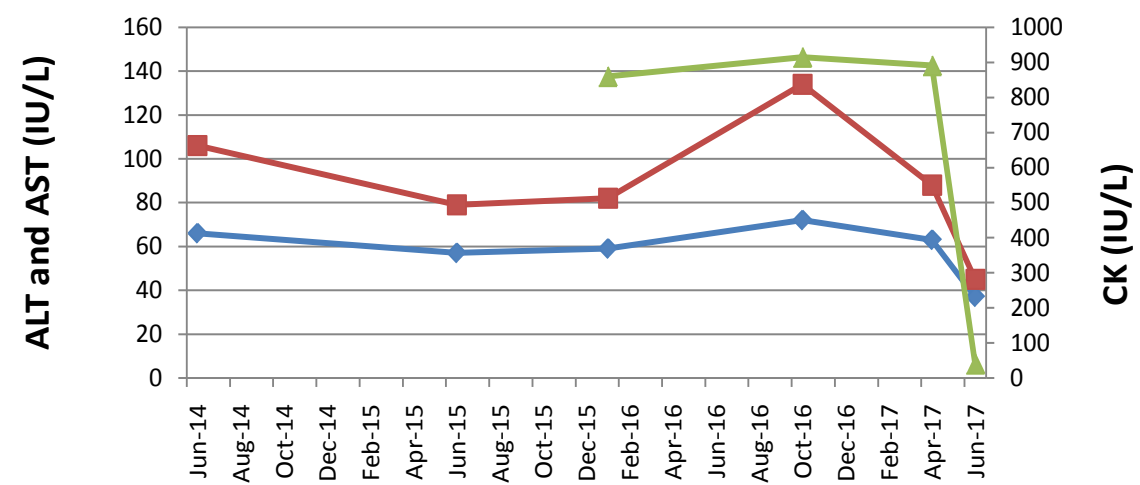

\begin{tabular}{|c|c|c|c|c|c|c|}
\cline { 2 - 7 } \multicolumn{1}{c|}{} & Jun-14 & Jun-15 & Jan-16 & Oct-16 & Apr-17 & Jun-17 \\
\hline$\sim$ ALT (IU/L) & 66 & 57 & 59 & 72 & 63 & 37 \\
\hline$\because-$ AST (IU/L) & 106 & 79 & 82 & 134 & 88 & 45 \\
\hline$\sim$ CK (IU/L) & & & 860 & 915 & 891 & 40 \\
\hline
\end{tabular}

Figure 2. Correlation of ALT and AST with CK over time showing a significant decline in enzymes on halting exercise. 
levels was done, this was ultimately proven to be a red herring. The liver biopsy was an essential part of the diagnostic pathway, since it ensured that our patient was not started on lifelong treatment with an antiviral agent.

Mild elevations in liver chemistry tests such as ALT and AST can reveal serious conditions or have transient and benign aetiologies. Potential causes for transaminitis include viral hepatitis, alcoholism, medications, steatosis or steatohepatitis, and cirrhosis. A good history should include a list of medications (including vitamins and herbs), alcohol, illicit drugs, exercise, family history and blood-product transfusions [1].

Elevations of ALT and AST are not exclusive to liver pathology. ALT and AST can be released by other organs in the body [2]. The American Gastroenterological Association (AGA) technical review states that serum ALT has diurnal variation, may vary day-to-day and may be affected by exercise or muscle injury [3].

AST is present in liver, muscle, heart, kidney, red blood cells, brain and small bowel; ALT is present in liver, muscle and kidney. Muscle actually has more AST and ALT when compared with liver because of large tissue mass [4]. If the elevated liver enzymes persist, other diagnostic tests would be appropriate. These could include ultrasonography, serum studies and potentially a liver biopsy.

In an article by Nathwani et al. in 2005, there seems to be two different thought processes with regards to muscle injury and elevated liver enzymes. Are the elevated liver enzymes a result of reversible damage being done to the liver or are they a result of these enzymes also being released from other solid body organs [5]?

The presence of a normal liver biopsy and normal FibroScan ${ }^{\circ}$ indicates that there was no ongoing reversible or irreversible damage to the liver. The presence of a high CK, which is confirmed to be released by skeletal muscle, indicates that the most likely source of these enzymes is muscle tissue. This was later on confirmed by the rapid decline in enzyme levels and CK levels once exercise was stopped.

Patients with muscular conditions such as polymyositis, statin-induced muscle injury and acute rhabdomyolysis have also been noted to have increased ALT and AST levels [5].

In a study by Pettersson et al. in 2008, AST and ALT were significantly increased for at least 7 days after the strenuous exercise, in this case weightlifting. The lactate dehydrogenase (LDH), CK and myoglobin were also elevated. In this study, healthy men who were used to moderate exercise, performed heavy weightlifting and their transaminases, $\mathrm{CK}, \mathrm{LDH}$ and myoglobin increased significantly after 1 hour of exercise. With this in mind, the authors highlighted the fact that it is important to restrict such strenuous exercise which can attribute to liver enzyme derangement when performing clinical studies [6].

Strength training and very heavy manual labour are more likely to cause raised transaminases than aerobic exercise. Transaminases can be elevated in marathon runners and they have the potential to develop rhabdomyolysis in extreme 
conditions [7].

This raises a strong argument for the inclusion of $\mathrm{CK}$ blood test in the screening panel and to inquire regarding exercise habits, since this might result in the prevention of inappropriate investigations, inappropriate discontinuation of medications or inappropriate referrals to specialist centres [6]. In the case of exercise-induced muscle injury, $\mathrm{CK}$ is transiently elevated but returns to normal levels along with transaminases after discontinuation of vigorous exercise. In patients with these elevated enzymes and in the absence of any risk factors, the importance of taking a full history including exercise habits cannot be understated.

\section{Funding}

No conflict of interest to declare.

\section{References}

[1] Giboney, P.T. (2005) Mildly Elevated Liver Transaminase Levels in the Asymptomatic Patient. American Family Physician, 71, 1105-1110.

[2] Giannini, E., Testa, R., Savarino, V., et al. (2005) Liver Enzyme Alteration: A Guide for Clinicians. CMAJ, 172, 367-379. https://doi.org/10.1503/cmaj.1040752

[3] Green, R.M. and Flamm, S. (2002) AGA Technical Review on the Evaluation of Liver Chemistry Tests. Gastroenterology, 123, 1367-1384. https://doi.org/10.1053/gast.2002.36061

[4] Pavletic, A.J., Pao, M. and Wright, M.E. (2015) Exercise-Induced Elevation of Liver Enzymes in a Healthy Female Research Volunteer. Psychosomatics, 56, 604-606. https://doi.org/10.1016/j.psym.2015.03.002

[5] Nathwani, R.A., Pais, S., Reynolds, T.B. and Kaplowitz, N. (2005) Serum Alanine Aminotransferase in Skeletal Muscle Diseases. Hepatology, 41, 380-382. https://doi.org/10.1002/hep.20548

[6] Petterson, J., Hindorf, U., Persson, P., Bengtsson, T., Malmqvist, U., Werkström, V. and Ekelund, M. (2007) Muscular Exercise Can Cause Highly Pathological Liver Function Tests in Healthy Men. British Journal of Clinical Pharmacology, 65, 253-259. https://doi.org/10.1111/j.1365-2125.2007.03001.x

[7] Sjogren, M.H. and Sjogren, M.H. (2007) Transaminase Levels and Vigorous Exercise. Gastroenterology \& Hepatology, 3, 913-914. 